\title{
Efeitos de um Programa de Reabilitação da Musculatura Inspiratória no Pós-Operatório de Cirurgia Cardíaca
}

\author{
Effects of an Inspiratory Muscle Rehabilitation Program in the Postoperative Period of Cardiac Surgery \\ Paulo Eduardo Gomes Ferreira1, Alfredo José Rodrigues ${ }^{1,2}$, Paulo Roberto Barboza Évora ${ }^{1,2}$ \\ Hospital das Clínicas da Faculdade de Medicina de Ribeirão Preto da Universidade de São Paulo ${ }^{1}$; Faculdade de Medicina da Universidade de \\ São Paulo de Ribeirão Preto², Ribeirão Preto, SP - Brasil
}

\section{Resumo}

Fundamento: Os músculos respiratórios são afetados pós operações cardíacas.

Objetivo: Verificar se o condicionamento pré-operatório dos músculos inspiratórios poderia ajudar a diminuir a disfunção respiratória pós-operatória.

Métodos: Trinta voluntários de ambos os sexos com idade mínima de 50 anos, aguardando cirurgia de revascularização do miocárdio e/ou cirurgia de válvula cardíaca foram alocados de forma randômica em dois grupos. Quinze pacientes foram incluídos em um programa domiciliar de pelo menos 2 semanas de treinamento pré-operatório dos músculos inspiratórios usando um dispositivo com uma carga correspondente a $\mathbf{4 0} \%$ da pressão inspiratória máxima. Os outros 15 receberam orientações gerais e não treinaram os músculos inspiratórios. A espirometria, antes e depois do programa de treinamento, bem como a evolução dos gases sanguíneos arteriais e das pressões inspiratória e expiratória máximas, foram avaliados em ambos os grupos antes e depois da cirurgia. Os desfechos clínicos dos dois grupos também foram comparados.

Resultados: Observamos que o treinamento dos músculos inspiratórios aumentou a capacidade vital forçada, a ventilação voluntária máxima e a relação entre o volume expirado forçado no primeiro segundo e a capacidade vital forçada. A evolução dos gases sanguíneos e das pressões expiratória e inspiratória máximas antes e depois da cirurgia foi similar em ambos os grupos, com desfechos também similares.

Conclusão: Concluímos que nosso programa domiciliar de treinamento dos músculos inspiratórios foi seguro e melhorou a capacidade vital forçada e a ventilação voluntária máxima, embora os benefícios clínicos desse programa não tenham sido claramente demonstrados no presente estudo. (Arq Bras Cardiol 2009;92(4):275-282)

Palavras-chave: Músculos respiratórios, cuidados pós operatórios, modalidades de fisioterapia, cirurgia torácica, reabilitação.

\section{Summary}

Background: Respiratory muscles are affected after cardiac surgeries.

Objective: To verify whether the preoperative conditioning of the inspiratory muscles might help to decrease postoperative respiratory dysfunction

Methods: Thirty volunteers of both genders and with a minimum age of 50 years, while waiting for myocardial revascularization and/or cardiac valve surgery, were randomly assigned to two groups. Fifteen patients were included in a domiciliary program of at least 2 weeks of preoperative training of the inspiratory muscles, using a device with a load corresponding to $40 \%$ of the maximum inspiratory pressure. The other 15 patients received general advice and did not train the inspiratory muscle. Spirometry, before and after the training program, as well as the evolution of the arterial blood gases and of the maximum inspiratory and expiratory pressure, before and after the operation were evaluated in both group. The clinical outcomes of the two groups were also compared.

Results: We observed that inspiratory muscle training increased the forced vital capacity, the maximum voluntary ventilation and the ratio between the forced expired volume during the first second and the forced vital capacity. The evolution of the arterial blood gases and of the maximum inspiratory and expiratory pressures before and after the operation was similar in both groups, with the outcomes also being similar.

Conclusion: We concluded that our domiciliary program of inspiratory muscle training was safe and improved the forced vital capacity and the maximum voluntary ventilation, although the clinical benefits of this program were not clearly demonstrable in the present study. (Arq Bras Cardiol 2009;92(4):261-268)

Key words: Respiratory muscles; thoracic surgery; postoperative care; physical therapy modalities; rehabilitation.

Full texts in English - http://www.arquivosonline.com.br

Correspondência: Paulo Eduardo Gomes Ferreira •

Av. Afonço Valera, 251/11, Recreio das Acácias - 14098-561 - Ribeirão Preto, SP - Brasil

E-mail: pegferreira@ig.com.br, pauloacdc@yahoo.com.br

Artigo recebido em 23/01/08; revisado recebido em 03/03/08; aceito em 06/03/08. 


\section{Introdução}

Complicações respiratórias estão entre as causas mais comuns de morbidade e mortalidade após cirurgia cardíaca13 . Disfunções respiratórias nos pós-operatório cardíaco são geralmente multifatoriais e alterações devido a cardiopatias ${ }^{4}$, à cirurgia em $\mathrm{si}^{5-7}$ e à limitações da reserva funcional pulmonar devido ao envelhecimento ${ }^{8,9}$ têm um papel preponderante. Vários estudos sugerem que, embora multifatorial, a morbidade e a mortalidade das causas pulmonares no pós-operatório poderiam ser reduzidas pelo treinamento da musculatura respiratória alterada por cardiopatias e envelhecimento ${ }^{10}$. Entretanto, esta é uma questão controversa e há poucos relatos sobre o papel do treinamento muscular respiratório na profilaxia das complicações respiratórias que surgem no pós-operatório cardíaco ${ }^{11-13}$

O objetivo desse estudo foi avaliar se o programa de treinamento pré-operatório para os músculos inspiratórios, conduzido em domicílio e planejado para melhorar as funções respiratórias, contribuiu para reduzir a morbidade e/ou mortalidade em pacientes adultos submetidos à cirurgia de revascularização do miocárdio e/ou valvuloplastia.

\section{Pacientes e Métodos}

A figura 1 mostra a linha do tempo das intervenções e a coleta de dados.

\section{Pacientes}

O estudo incluiu 30 pacientes voluntários de ambos os sexos, sem discriminação de etnia, com menos de 50 anos de idade, que foram submetidos à cirurgia de revascularização miocárdica ou valvuloplastia. Todos os pacientes assinaram o termo de consentimento livre e informado e o estudo foi aprovado pelo Comitê de Ética em Pesquisa do Hospital da Faculdade de Medicina de Ribeirão Preto, Universidade de São Paulo (HCFMRP-USP).

Os pacientes foram aleatoriamente divididos em dois grupos, Grupo Controle (GC) e Grupo de Incentivo
Respiratório (IR). Ambos os grupos receberam informações gerais, mas apenas os indivíduos do grupo IR foram submetidos ao incentivo respiratório.

Os critérios de exclusão foram: pacientes com angina instável no momento da seleção ou durante o programa, insuficiência cardíaca congestiva descompensada, falta de capacidade física ou intelectual de executar os exercícios prescritos, arritmia ventricular complexa e não-controlada, pressão arterial não-controlada (>140/90 mmHg), infarto do miocárdio ou acidente vascular cerebral (AVC) há menos de 3 anos, bloqueio átrio-ventricular de alto grau ou broncoespasmo induzido por exercício. Os pacientes submetidos à procedimentos cirúrgicos antes de completar um mínimo de 2 semanas de treinamento muscular respiratório também foram excluídos.

O programa foi descontinuado para pacientes que reclamaram de dispnéia, antes ou depois do exercício; desconforto abdominal ou pré-cordial ou dor, ou qualquer outro sintoma auto-caracterizado como "anginoso"; tontura, escurecimento da visão ou qualquer outro tipo de desconforto físico ou mental; vômitos ou náusea; taquicardia ou bradicardia; cansaço que evitasse a continuação do exercício; cianose ou palidez da pele ou mucosa; vertigem, tontura ou perda de consciência.

\section{Procedimentos fisioterapêuticos}

Os pacientes em ambos os grupos foram submetidos aos seguintes procedimentos: a) Manovacuometria; b) Espirometria; c) Coleta de sangue arterial para gasometria.

\section{Manovacuometria}

Para avaliações através desse método, as pressões de pico inspiratória e expiratória, pressão inspiratória máxima (PIMax) e pressão expiratória máxima (PEMax), respectivamente, foram determinadas pelo volume residual (VR) e capacidade pulmonar total $(\mathrm{CPT})^{14}$, medidas com um manovacuômetro, modelo MV-150/300 (Ger-Ar Comércio Equipamentos Ltda.

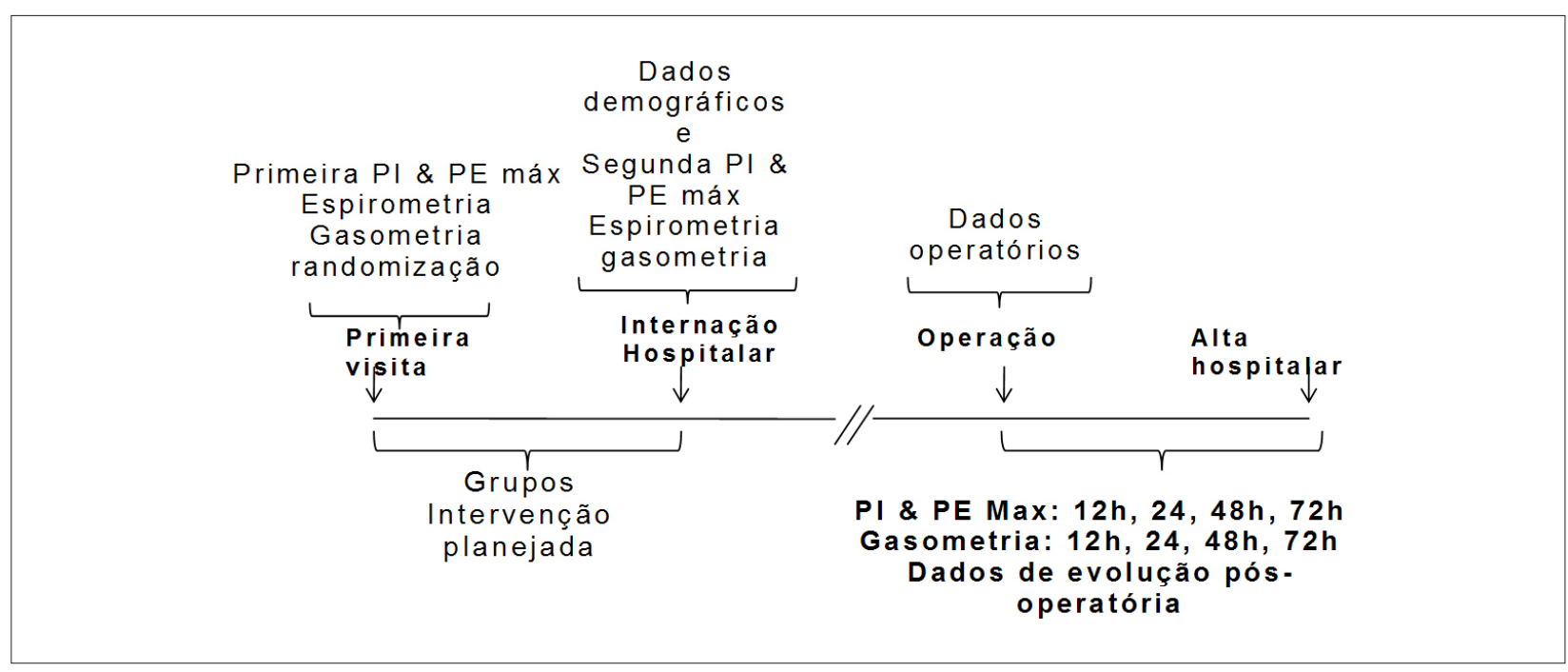

Fig. 1 - Linha do tempo das intervenções e coleta de dados. 
São Paulo, Brasil). Três medidas consecutivas, tecnicamente aceitáveis, foram feitas com intervalos mínimos de 1 minuto. As mensurações que produziram valores com diferenças acima de $10 \%$ foram descartadas e novas medidas foram repetidas a fim de obter três medidas adequadas ${ }^{14}$. A primeira verificação do PIMax e PEMax (D0) foi conduzido no início do programa, o segundo logo após a internação para a cirurgia programada (DInt) e os outros 12, 24, 48 e 72 horas (12h PC, 24 PC, 48 PC e 72 PC, respectivamente) após a extubação pós-operatória. Durante todas as medidas pós-operatórias, os pacientes permaneciam sentados na cama e usando um clipe nasal.

\section{Testes de função pulmonar}

Ambos os grupos de pacientes foram submetidos a testes de espirometria no início do programa (D0) e à internação, imediatamente antes da cirurgia (DInt). Todas as medidas espirométricas foram realizadas no Laboratório de Função Pulmonar no Hospital Universitário da Faculdade de Medicina de Ribeirão Preto, Universidade de São Paulo (HCFMRP-USP), pelo mesmo profissional, à temperatura ambiente constante $\left(22^{\circ} \mathrm{C}\right)$ usando um espirômetro de 9,0 litros, modelo GS Plus (Collins, EUA).

\section{Gasometria arterial}

As amostras de sangue foram coletadas no primeiro teste de espirometria (D0) antes do programa de exercícios e novamente na internação (DInt). Outras amostras foram coletadas após a cirurgia, respectivamente, logo após a extubação orotraqueal e 12, 24, 48 e 72 horas após a extubação pós-operatória.

\section{Orientações gerais para a pré-cirurgia}

Pacientes em ambos os grupos, GC e IR, receberam orientações sobre a cirurgia e características pós-operatórias. Eles foram informados sobre os efeitos danosos do fumo e foram estimulados a não fumar antes da cirurgia. Exercícios de inspiração profunda sem equipamento especial e caminhadas diárias até o próprio limite ser atingido foram também sugeridos aos pacientes. Os mesmos procedimentos fisioterapêuticos pós-operatórios foram empregados em todos os pacientes em ambos os grupos, de acordo com a necessidade e programas de rotina estabelecidos pelo Setor de Fisioterapia da Divisão de Cirurgia Cardiovascular e Torácica do HCFMRP-USP.

\section{Programa de treinamento para a musculatura inspiratória}

Os pacientes foram instruídos a fazer cinco séries de 10 inspirações profundas e calmas, com um intervalo de pelo menos 1 minuto entre as séries, sem se cansarem e sem se sentirem enjoados, com o incentivo de um instrumento respiratório, "Threshold IMT" (Respironics, Cedar Grove, NJ, EUA), com uma carga de $40 \%$ da PIMax (D0) ${ }^{15}$. As séries deveriam ser repetidas três vezes ao dia durante o período de espera pela cirurgia.

\section{Análise estatística}

Todos os valores foram expressos como médias \pm desviospadrão ou porcentagens. O teste de Shapiro-Wilks foi utilizado para determinar distribuições normais. O teste $t$ de Student para valores pareados e não-pareados, como indicado, foi utilizado quando as variáveis apresentavam distribuição normal e eram contínuas. Caso contrário, os testes de MannWhitney ou de Wilcoxon eram utilizados. O teste exato de Fisher foi usado para comparar proporções. O teste "TwoWay ANOVA" foi usado para comparar três ou mais medidas repetidas, intra- e intergrupos.

\section{Resultados}

Nenhum paciente precisou abandonar o programa devido à eventos indesejáveis. Não houve mortes durante o programa; um paciente deixou o programa voluntariamente e nenhum paciente foi excluído para antecipação da cirurgia antes de pelo menos 15 dias de participação no programa. Não houve valores perdidos para quaisquer dados coletados.

\section{Dados demográficos}

A Tabela 1 mostra os dados demográficos de pacientes de ambos os grupos. Exceto por uma prevalência de pacientes do sexo masculino no grupo controle $(p=0,007)$ não houve outras diferenças significantes entre os grupos.

\section{Dados operatórios}

A duração do uso do clampe de aorta foi significantemente maior no grupo IR $(92,07 \pm 31,03$ vs $73,5 \pm 26,98 ; p=0,034)$. Não houve diferenças nos períodos de circulação extracorpórea $(111,6 \pm 74,06$ vs $112,3 \pm 4,5 ; p=0,696)$ e no número de anastomoses distais na revascularização $(2,8 \pm 0,77$ vs $3,0 \pm 0,87 ; p=0,656)$. A revascularização do miocárdio foi realizada em $73,3 \%$ dos pacientes do grupo controle e em $60,0 \%$ dos pacientes do grupo IR $(p=0,700)$.

\section{Função respiratória}

No grupo IR, o período de tempo entre a inclusão do paciente no programa e a internação para cirurgia, isto é, o tempo disponível para o treinamento da musculatura respiratória, foi $154,0 \pm 87,4$ dias.

\section{Espirometria}

A Tabela 2 mostra os valores de espirometria para o grupo controle no início do programa, na internação e antes da cirurgia. A comparação dos valores espirométricos no grupo IR, antes e depois do período de treinamento, mostra aumentos significantes, ambos em valores absolutos e na porcentagem da capacidade vital forçada (CVF) preditiva. O mesmo foi observado em relação a valores absolutos da ventilação voluntária máxima (VMM), mas sem elevação significante na porcentagem preditiva. Houve uma diminuição significante na relação VEF1/CVF, já que a CVF estava aumentada e o volume expiratório forçado após 1 minuto (VEF1) não mudou. Entretanto, a diminuição não foi significante quando comparada ao percentil previsto da relação VEF1/CVF.

\section{Manovacuometria}

Não houve diferença significante entre os grupos em 
Tabela 1 - Dados demográficos de pacientes nos grupos controle (GC) e incentivo respiratório (IR). Dados expressos como médias \pm DP ou porcentagens

\begin{tabular}{|c|c|c|c|}
\hline & $\begin{array}{c}\mathrm{GC} \\
\mathrm{n}=15\end{array}$ & $\begin{array}{c}\text { IR } \\
n=15\end{array}$ & $P$ \\
\hline \multicolumn{4}{|l|}{ Sexo } \\
\hline Masculino & $13(86,7 \%)$ & $9(60 \%)$ & \multirow{2}{*}{0,007} \\
\hline Feminino & $2(13,3 \%)$ & $6(40 \%)$ & \\
\hline Idade (anos) & $63,07 \pm 7,93$ & $62,47 \pm 6,06$ & 0,818 \\
\hline IMC & $28,34 \pm 3,16$ & $26,60 \pm 5,17$ & 0,815 \\
\hline Fumante & $1(6,7 \%)$ & $3(20 \%)$ & 0,598 \\
\hline Ex-fumante & $4(26,7 \%)$ & $4(26,7 \%)$ & 1,000 \\
\hline \multicolumn{4}{|l|}{ Classe Funcional (NYHA) } \\
\hline Classe I & $10(66,7 \%)$ & $7(46,7 \%)$ & 0,462 \\
\hline Classe II & $3(20 \%)$ & $5(33,3 \%)$ & 0,682 \\
\hline Classe III & $2(13,3 \%)$ & $2(13,3 \%)$ & 1,000 \\
\hline Classe IV & $0(0 \%)$ & $1(6,7 \%)$ & 1,000 \\
\hline $\mathrm{FE}<35$ & $0(0 \%)$ & $2(13,3 \%)$ & 0,483 \\
\hline Fibrilação atrial crônica & $3(20 \%)$ & $3(20 \%)$ & 1,000 \\
\hline Hipertensão arterial & $10(66,7 \%)$ & $10(66,7 \%)$ & 1,000 \\
\hline Insuficiência cardíaca & 0 & $1(6,7 \%)$ & 1,000 \\
\hline Infarto do miocárdio prévio & $7(46,7 \%)$ & $4(26,7 \%)$ & 0,256 \\
\hline $\begin{array}{l}\text { Pressão sistólica pulmonar } \\
>40 \mathrm{mmHg}\end{array}$ & 0 & $3(20 \%)$ & 0,224 \\
\hline Diabetes mellitus & $6(40 \%)$ & $6(40 \%)$ & 1,000 \\
\hline Obstrução da artéria carótida & 0 & $1(6,7 \%)$ & 1,000 \\
\hline Doença pulmonar obstrutiva & $1(6,7 \%)$ & $1(6,7 \%)$ & 1,000 \\
\hline \multicolumn{4}{|l|}{ Doença pulmonar restritiva } \\
\hline Leve & 0 & $1(6,7 \%)$ & 1,000 \\
\hline Moderada & 0 & $1(6,7 \%)$ & 1,000 \\
\hline Acidente vascular cerebral & 0 & $2(13,3 \%)$ & 0,483 \\
\hline Doença vascular periférica & 0 & $1(6,7 \%)$ & 0,215 \\
\hline
\end{tabular}

relação à PIMax e PEMax. Entretanto, em ambos os grupos, a PIMax estava significantemente aumentada, quando comparados os valores à internação e à primeira mensuração, D0, considerado como controle em cada grupo (Figura 2). Todas as mensurações de PIMax pós-operatórias foram significantemente mais baixas $(p<0,001)$. A evolução da PEMax foi similar à da PIMax, como demonstrado na Figura 3, exceto que não houve diferença entre a primeira mensuração (D0) e aquela realizada no dia da internação (DInt). Entretanto, a diminuição foi significante nas mensurações pós-operatórias quando comparadas a ao D0 $(p<0,001)$.

\section{Gasometria}

A Figura 4 mostra que a evolução temporal do PO2 arterial não foi diferente entre os dois grupos. Houve um aumento significante de PO2 ( $p<0,001)$ em ambos os grupos, imediatamente após a extubação, quando comparado com o D0, devido à suplementação de oxigênio de rotina em todos os pacientes nesse momento. Entretanto, os valores voltaram aos níveis similares ao D0 em mensurações posteriores durante o período pós-operatório. A evolução temporal do PCO2 mostrada na Figura 5 não indica diferenças entre os grupos. Além disso, os valores de PCO2 arterial mantiveram-se na faixa normal e desenvolveram-se de forma similar. Em ambos os grupos, uma diminuição significante de PCO2 arterial foi evidenciada após 48 e 72 horas, em comparação com D0, mas os valores ainda estavam dentro da faixa de normalidade.

\section{Evolução clínica pós-operatória}

A Tabela 3 mostra a incidência de complicações e taxas de mortalidade pós-operatória em cada grupo. Embora as diferenças não fossem significantes, houve quatro mortes (em até 30 dias após a alta hospitalar). Dessas, 3 mortes ocorreram no grupo IR: uma devido a trombose aorto-ilíaca, uma devido a morte súbita de causa não revelada e uma terceira causada por pneumonia pós-operatória. A única morte no grupo controle foi causada por insuficiência cardíaca refratária no pós-operatório imediato. Todos esses pacientes apresentavam disfunção ventricular esquerda no período pré-operatório (fração de ejeção $\leq 0.35$ e/ou Classe Funcional III/IV da NYHA).

\section{Discussão}

Embora o treinamento muscular inspiratório pré-operatório não tenha mostrado benefícios clínicos aparentes, ele aumentou, de forma significante, a função ventilatória, como demonstrado pelo aumento da capacidade vital forçada e a ventilação voluntária máxima.

Como esperado, o treinamento da musculatura inspiratória não influenciou a PEMax. Entretanto, o aumento da PIMax foi significante no dia da internação em comparação com o primeiro valor determinado em ambos os grupos. Embora o único grupo que mostrou benefícios respiratórios tenha sido o que recebeu treinamento, como demonstrado pelos valores da espirometria na internação, a melhora na PIMax, independente do treinamento, sugere que o conhecimento metodológico prévio pelos pacientes pode ter contribuído para as melhores condições de teste na internação. Resultados similares foram relatados por Newall e cols. ${ }^{16}$ em seus pacientes-controle, embora sua argumentação tenha sido de que os exercícios de alta intensidade sem carga conduzidos pelos pacientes-controle poderiam ter melhorado a função inspiratória expressa pela alta PIMax também nesse grupo.

No presente estudo, o grupo controle não recebeu um treinamento específico da musculatura inspiratória, mas foram igualmente aconselhados a parar de fumar e fazer exercícios de caminhada diários, além de receberem instruções para realizar exercícios de respiração profunda na proporção de 3 séries de 10 repetições, diariamente, sem equipamento.

É possível que essas medidas e exercícios, mesmo sem carga, tenham contribuído para melhorar a PIMax no grupo controle. A falta de alterações nos valores espirométricos nesse 
Tabela 2 - Valores da espirometria para ambos os grupos de pacientes, GC e IR, no inicio do programa (D0) e no dia da internação hospitalar (DInt). Os valores são expressos como médias \pm DP. 0 teste $t$ de Student pareado foi utilizado para comparar D0 e DInt para cada grupo

\begin{tabular}{|c|c|c|c|c|c|c|}
\hline & \multicolumn{3}{|c|}{ Grupo Controle (GC) } & \multicolumn{3}{|c|}{ Grupo Incentivo Respiratório (IR) } \\
\hline & Do & Dint & $\mathrm{p}$ & Do & Dint & $\mathrm{p}$ \\
\hline CVF & $3,2 \pm 0,7$ & $3,2 \pm 0,74$ & 0,843 & $2,9 \pm 1,14$ & $3,1 \pm 1,6$ & 0,006 \\
\hline CVF (\% predito) & $88,1 \pm 13,8$ & $92,1 \pm 23,73$ & 0,480 & $86,3 \pm 21,06$ & $95,7 \pm 21,5$ & 0,002 \\
\hline CRF & $3,3 \pm 0,8$ & $3,5 \pm 0,82$ & 0,369 & $3,5 \pm 1,03$ & $3,3 \pm 1,4$ & 0,307 \\
\hline CRF (\% predita) & $104,8 \pm 25,9$ & $111,6 \pm 22,36$ & 0,235 & $115,3 \pm 23,69$ & $105,9 \pm 31,7$ & 0,230 \\
\hline VR & $2,8 \pm 0,7$ & $3,0 \pm 0,78$ & 0,548 & $2,7 \pm 0,51$ & $2,4 \pm 0,8$ & 0,077 \\
\hline VR (\% predito) & $143,4 \pm 36,0$ & $148,4 \pm 29,99$ & 0,571 & $144,2 \pm 22,04$ & $109,3 \pm 51,3$ & 0,079 \\
\hline СРT & $6,1 \pm 1,4$ & $6,23 \pm 1,36$ & 0,364 & $5,8 \pm 1,57$ & $5,6 \pm 1,8$ & 0,208 \\
\hline CPT (\% predita) & $106,8 \pm 19,4$ & $112,1 \pm 19,15$ & 0,224 & $107,6 \pm 20,7$ & $100,8 \pm 19,5$ & 0,157 \\
\hline VVM & $79,4 \pm 24,5$ & $72,9 \pm 30,37$ & 0,278 & $76,0 \pm 34,37$ & $83,1 \pm 35,9$ & 0,044 \\
\hline VVM (\% predita) & $75,5 \pm 18,8$ & $70,1 \pm 22,42$ & 0,343 & $73,9 \pm 22,72$ & $81,8 \pm 23,4$ & 0,075 \\
\hline VEF1 & $2,4 \pm 0,6$ & $2,4 \pm 0,61$ & 0,800 & $2,3 \pm 0,89$ & $2,4 \pm 0,9$ & 0,453 \\
\hline VEF1 (\% predita) & $92,3 \pm 17,1$ & $93,3 \pm 21,19$ & 0,743 & $92,9 \pm 22,29$ & $97,7 \pm 23,0$ & 0,246 \\
\hline VEF1/FVC & $75,1 \pm 9,6$ & $73,8 \pm 9,56$ & 0,460 & $79,7 \pm 5,68$ & $76,2 \pm 6,2$ & 0,008 \\
\hline FEV1/FVC (\% predita) & $103,4 \pm 14$ & $103,0 \pm 12,04$ & 0,925 & $109,0 \pm 10,07$ & $102,9 \pm 11,7$ & 0,097 \\
\hline FEF $25-75 \%$ & $2,1 \pm 1,0$ & $2,1 \pm 1,06$ & 0,817 & $2,4 \pm 1,14$ & $2,2 \pm 1,3$ & 0,216 \\
\hline FEF $25-75 \%$ (\% predita) & $78,6 \pm 38,3$ & $75,7 \pm 33,16$ & 0,720 & $88,6 \pm 34,45$ & $83,0 \pm 40,2$ & 0,377 \\
\hline FEF $50 \%$ & $2,6 \pm 1,7$ & $2,5 \pm 1,35$ & 0,777 & $3,5 \pm 1,56$ & $3,4 \pm 1,8$ & 0,750 \\
\hline FEF 50\% (\% predita) & $57,1 \pm 36,6$ & $55,0 \pm 28,11$ & 0,815 & $71,5 \pm 28,96$ & $72,0 \pm 36,7$ & 0,951 \\
\hline FEF $75 \%$ & $0,74 \pm 0,4$ & $0,68 \pm 0,34$ & 0,427 & $1 \pm 0,47$ & $0,94 \pm 0,48$ & 0,532 \\
\hline FEF 75\% (\% predita) & $43,6 \pm 22,3$ & $41,45 \pm 20,74$ & 0,623 & $50,44 \pm 22,4$ & $49,66 \pm 24,8$ & 0,861 \\
\hline
\end{tabular}

CVF - capacidade vital forçada; CRF - capacidade residual funcional; VR - volume residual; CPT - capacidade pulmonar total; VVM - ventilação voluntária máxima; VEF1 - volume expiratório forçado no primeiro segundo; FEF - fluxo expiratório forçado.

grupo reforça a hipótese de que o conhecimento prévio da técnica de manovacuometria tenha influenciado a obtenção dos valores de PIMax.

Os efeitos positivos do treinamento da musculatura inspiratória na ventilação pulmonar foram demonstrados pelo aumento na capacidade vital forçada (CVF) e ventilação voluntária máxima (VVM), embora os benefícios clínicos não tivessem sido aparentes. Entretanto, devido ao fato de vários pacientes voluntários não apresentarem disfunção pulmonar, tornou-se difícil demonstrar os benefícios clínicos. Não obstante, outros estudos ${ }^{11,12,17}$ sugeriram o treinamento pré-operatório como medida preventiva de complicações respiratórias, bem como em situações clínicas, como insuficiência cardíaca, melhora na sensação de dispnéia, possibilidade de aumento de distâncias caminhadas e melhor qualidade de vida ${ }^{18}$.

Um aspecto do programa que não pode ser controlado pelos organizadores, é o período de tempo do inicio do programa até a internação para realização da cirurgia. Como em muitas outras instituições brasileiras, a demanda por cirurgias cardíacas pagas pelo Sistema Único de Saúde (SUS) é muito alta, resultando em longos períodos de espera e diminuição da motivação por parte dos pacientes. Alguns autores consideram ${ }^{16,18}$ que os efeitos do treinamento respiratório podem persistir por várias semanas após seu término e isso pode explicar os resultados positivos da espirometria em uma população cuja aderência ao programa diminuiu com o tempo.

A significante redução pós-operatória no desempenho da musculatura respiratória era esperada, como demonstrada pelos valores mais baixos de PEMax e PIMax em ambos os grupos. Dor, os efeitos da cirurgia cardíaca na função muscular e o efeito dos analgésicos provavelmente contribuem para esses achados. Como mencionado anteriormente, os altos níveis de PO2 na extubação foram provavelmente devidos à suplementação de oxigênio nas primeiras 12 horas após a cirurgia. A redução no PCO2 arterial observada 48 e 72 horas após a cirurgia não tem valor clínico, estando dentro da normalidade.

O treinamento respiratório perioperatório é considerado benéfico por vários cirurgiões e fisioterapeutas especializados em pós-cirurgia, embora não haja um consenso sobre sua eficácia profilática. Diversamente, Pasquina e cols. ${ }^{19}$, em uma revisão recente, concluiu que a fisioterapia respiratória profilática não é efetiva na prevenção de complicações 


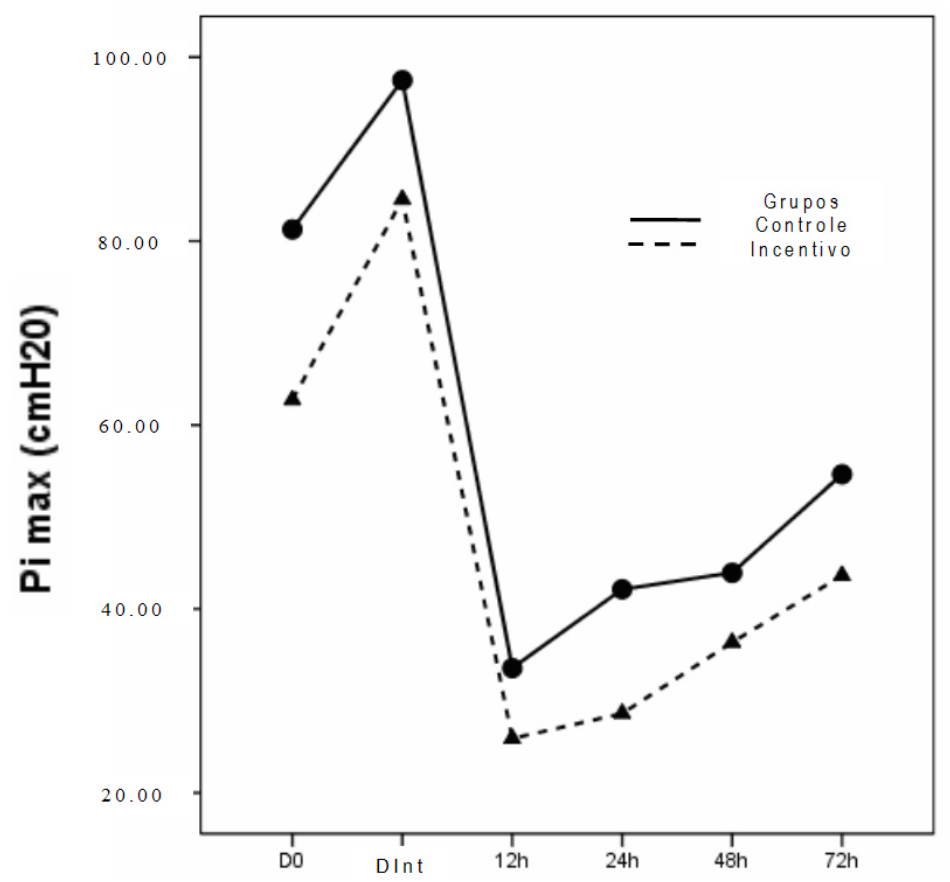

Fig. 2 - Variações no pico de pressão inspiratória (PI Max) no início do programa (D0), no dia da internação e 12, 24, 48 e 72 horas pós-operatórias em ambos os grupos GC e IR; * diferença significante $(p<0,001)$ em relação ao D0 no mesmo grupo.

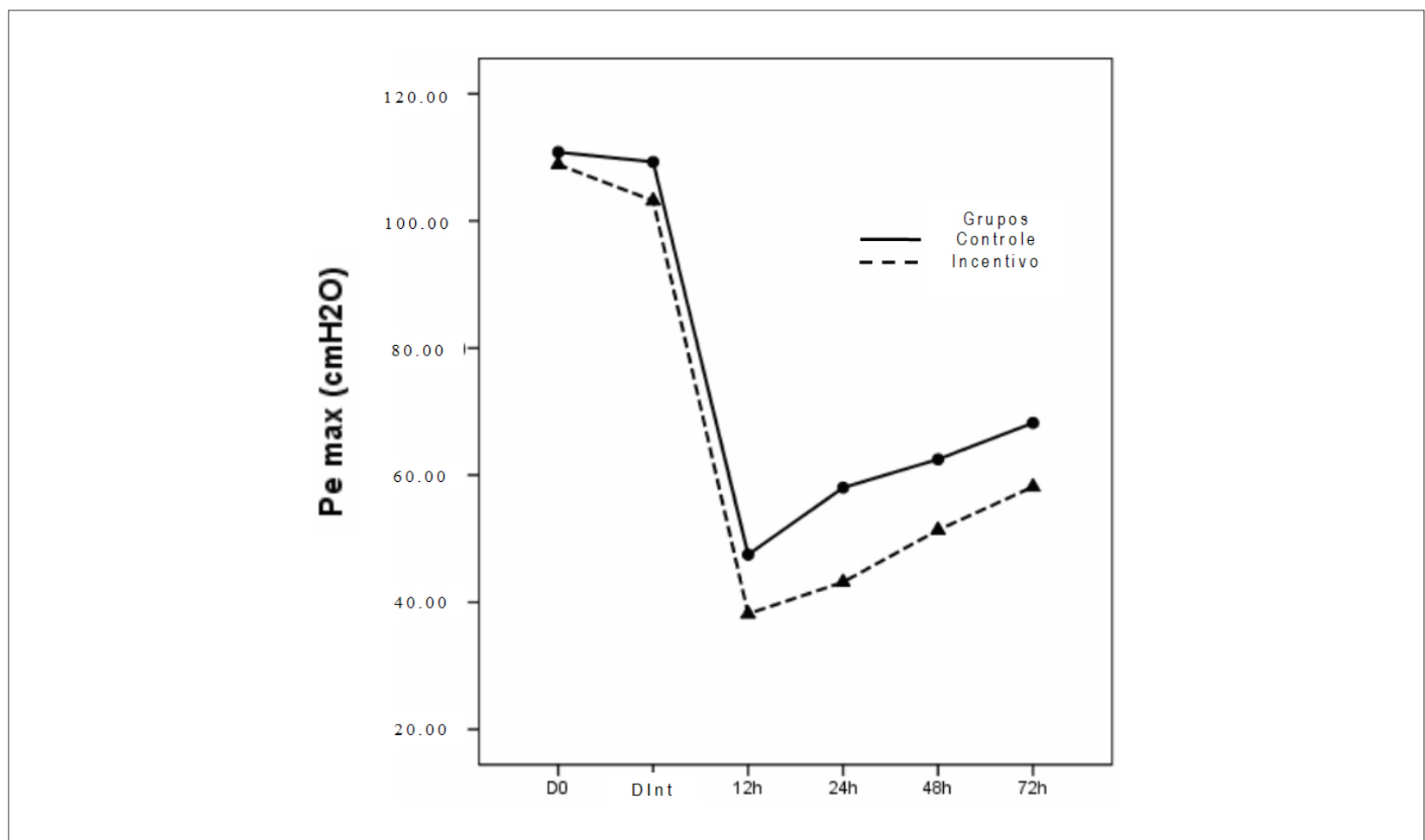

Fig. 3 - Variações no pico de pressão expiratória (PE Max) no início do programa (DO), no dia da internação e 12, 24, 48 e 72 horas pós-operatórias em ambos os grupos GC e IR; * diferença significante $(p<0,001)$ em relação ao D0 no mesmo grupo. 


\section{Artigo Original}

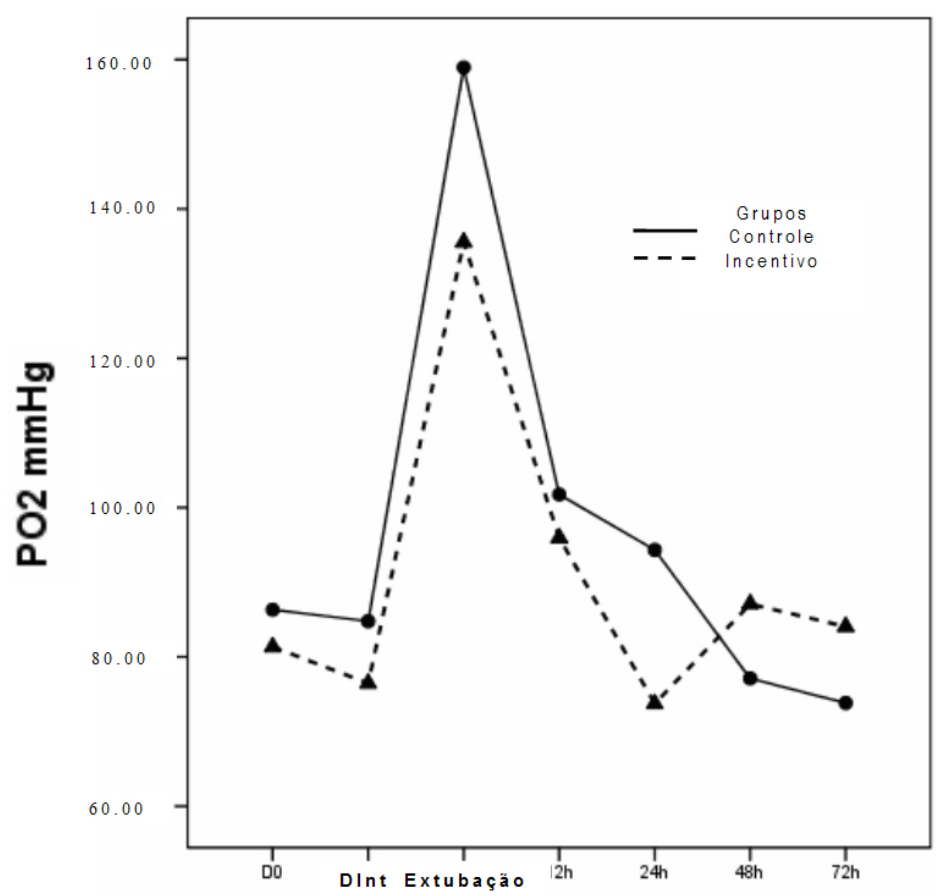

Fig. 4 - Evolução temporal de PO2 no início do programa (D0), no dia da internação (DInt), logo após a extubação orotraqueal e 12, 24, 48 e 72 horas pós-operatórias em ambos os grupos, controle (GC) e incentivo respiratório (IR). * diferença significante $(p<0,001)$ em relação ao D0 no mesmo grupo.

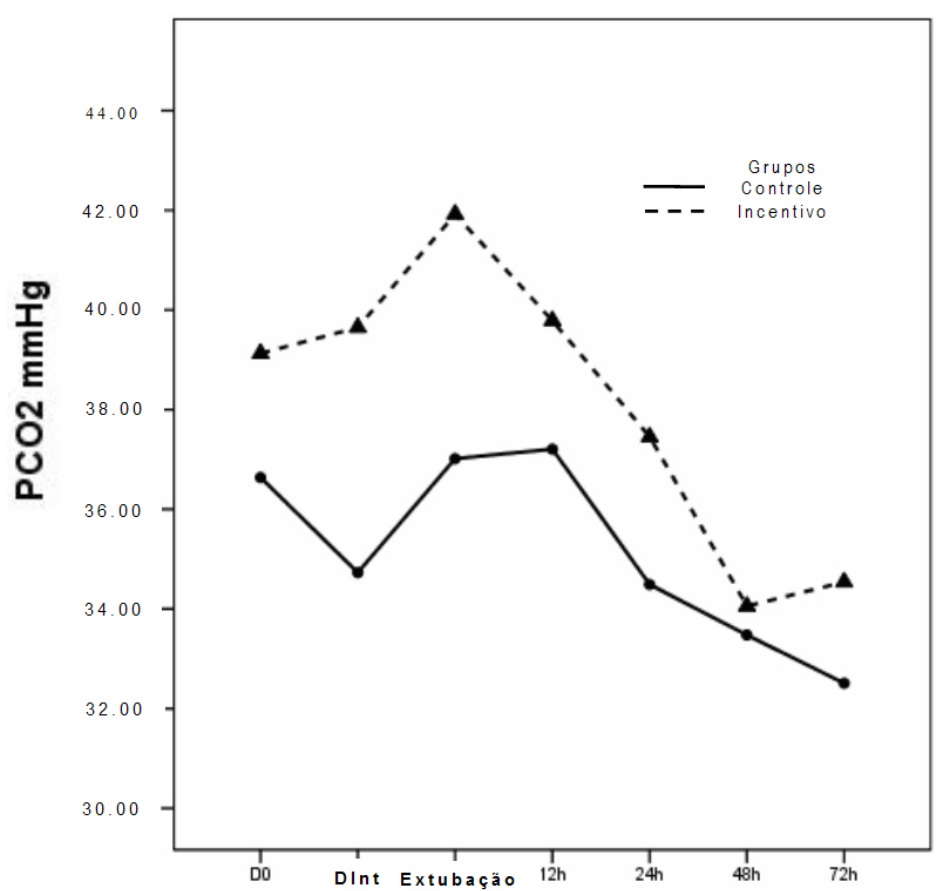

Fig. 5 - Evolução temporal de PCO2 no início do programa (D0), no dia da internação (DInt), logo após a extubação orotraqueal e 12, 24, 48 e 72 horas pós-operatórias em ambos os grupos, controle (GC) e incentivo respiratório (IR) . * diferença significante $(p<0,001)$ em relação ao $D 0$ no mesmo grupo. 
Tabela 3 - Evolução clínica pós-operatória

\begin{tabular}{lccc}
\hline & \multicolumn{2}{c}{ Grupos } & P \\
\cline { 2 - 3 } & $\begin{array}{c}\text { GC } \\
\mathrm{n}=\mathbf{1 5}\end{array}$ & $\begin{array}{c}\mathbf{I R} \\
\mathrm{n}=15\end{array}$ & \\
\hline Pneumonia & 0 & $1(6,7 \%)$ & 1,000 \\
\hline $\begin{array}{l}\text { Ventilação prolongada } \\
(>48 \mathrm{~h})\end{array}$ & 0 & $1(6,7 \%)$ & 1,000 \\
\hline Insuficiência renal & 0 & $3(20 \%)$ & 0,224 \\
\hline Insuficiência cardíaca & $1(6,7 \%)$ & $1(6,7 \%)$ & 1,000 \\
\hline Mortes & $1(6,7 \%)$ & $3(20 \%)$ & 0,598 \\
\hline
\end{tabular}

pulmonares pós-cirurgia cardíaca. Além de criticar a qualidade de alguns dos estudos, os autores consideraram que não há evidencias suficientes para garantir a utilidade desses procedimentos. Dessa forma, estudos prospectivos sobre o caráter profilático da fisioterapia respiratória são necessários. O estudo prospectivo de Leguisamo e cols. ${ }^{20}$ observaram que um programa pré-operatório de fisioterapia resultou em redução significante da estadia hospitalar ${ }^{20}$.

Nosso estudo, embora prospectivo com escolha aleatória de voluntários, não está livre de críticas. O tamanho da amostra é pequeno, especialmente se considerarmos os dois tipos de cirurgia cardíaca. Além disso, a falta de monitoração rigorosa pode ter comprometido a eficácia do programa,

\section{Referências}

1. Silva LHF, Nascimento CS, Viotti Jr LAP. Revascularização do miocárdio em idosos. Rev Bras Cir Cardiovasc. 1997; 12 (2): 132-40.

2. Rodriguez R, Torrents A, Garcia P, Ribera A, Permanyer G, Moradi M, et al. Cardiac surgery in elderly patients. Rev Esp Cardiol. 2002; 55 (11): 1159-68.

3. Jin F, Chung F. Minimizing perioperative adverse events in the elderly. $\mathrm{Br}$ J Anaesth. 2001; 87 (4): 608-24.

4. Meyer FJ, Borst MM, Zugck C, Kirschke A, Schellberg D, Kubler W, et al. Respiratory muscle dysfunction in congestive heart failure: clinical correlation and prognostic significance. Circulation. 2001; 103: 2153-8.

5. Matthay MA, Wiener-Kronish JP. Respiratory management after cardiac surgery. Chest. 1989; 95 (2): 424-34.

6. Diegeler A, Doll N, Rauch T, Haberer D, Walther T, Falk V, et al. Humoral immune response during coronary artery bypass grafting: a comparison o limited approach, "off-pump" technique, and conventional cardiopulmonary bypass. Circulation. 2000; 102 (Suppl 3): III 95-III 100

7. Singh NP, Vargas FS, Cukier A. Arterial blood gases after coronary artery bypass surgery. Chest. 1992; 102: 1337-41.

8. Johnson BD, Reddan WG, Pegelow DF, Seow KC, Dempsey JA. Flow limitation and regulation of functional residual capacity during exercise in a physically active aging population. Am Rev Respir Dis. 1991; 143 (5): 960-7.

9. Shephard RJ. Exercise and aging: extending independence in older adults. Geriatrics. 1993; 48 (5): 61-4.

10. Tolep K, Kelsen SG. Effect of aging on respiratory skeletal muscles. Clin Chest Med. 1993; 14 (3): 363-78

11. Weiner P, Zeidan F, Zamir D, Pelled B, Waizman J, Beckerman M, et al. Prophylactic inspiratory muscle training in patients undergoing coronary prejudicando seus benefícios clínicos.

O fato de que apenas um tipo de intervenção e uma modalidade de exercício respiratório foram utilizados, é outra característica possível de ser criticada.

Se a melhora de alguns parâmetros espirométricos vista após o treinamento inspiratório será transformada em benefícios clínicos, é uma questão que exige outros estudos clínicos com um número consideravelmente maior de pacientes.

Em conclusão, o estudo mostrou que o programa de treinamento muscular inspiratório pode ser conduzido em casa, é seguro e resultou na melhora da capacidade vital forçada e ventilação voluntária máxima, embora seus benefícios clínicos não sejam evidentes.

\section{Potencial Conflito de Interesses}

Declaro não haver conflito de interesses pertinentes.

\section{Fontes de Financiamento}

O presente estudo não teve fontes de financiamento externas.

\section{Vinculação Acadêmica}

Este artigo é parte de tese de Mestrado de Paulo Eduardo Gomes Ferreira pela Faculdade de Medicina de Ribeirão Preto da Universidade de São Paulo. artery bypass graft. World J Surg. 1998; 22: 427-31.

12. Weiner P, Man A, Weiner M, Rabner M, Waizman J, Magadle R, et al. The effect of incentive spirometry and inspiratory muscle training on pulmonary function after lung resection. J Thorac Cardiovasc Surg. 1997; 113 (3): 552-7.

13. Nomori H, Kobayashi R, Fuyuno G, Morinaga S, Yashima H. Preoperative respiratory muscle training: assessment in thoracic surgery patients with special reference to postoperative pulmonary complications. Chest. 1994; 105 (6): 1782-8.

14. Souza RB. Pressões respiratórias estáticas máximas. J Bras Pneumol. 2002 28 (supl. 3): 155-65.

15. Sociedade Brasileira de Pneumologia e Tisiologia. Consenso Brasileiro de Ventilação mecânica. J Bras Pneumol. 2000; 26 (supl 2): S1-S68.

16. Newall C, Stockley RA, Hill SL. Exercise training and inspiratory muscle training in patients with bronchiectasis. Thorax. 2005; 60 (11): 943-8.

17. Rajendran AJ, Pandurangi UM, Murali R, Gomathi S, Vijayan VK, Cherian KM Pre-operative short-term pulmonary rehabilitation for patients of chronic obstructive pulmonary disease undergoing coronary artery bypass graft surgery. Indian Heart J. 1998; 50 (5): 531-4.

18. Dall'Ago P, Chiappa GR, Guths H, Stein R, Ribeiro JP. Inspiratory muscle training in patients with heart failure and inspiratory muscle weakness: a randomized trial. J Am Coll Cardiol. 2006; 47 (4): 757-63.

19. Pasquina P, Tramer MR, Walder B. Prophylactic respiratory physiotherapy after cardiac surgery: systematic review. BMJ. 2003; 327: 1-6.

20. Leguisamo CP, Kalil RA, Furlani AP. A efetividade de uma proposta fisioterapêutica pré-operatória para cirurgia de revascularização do miocárdio. Rev Bras Cir Cardiovasc. 2005; 20 (2): 134-41. 